\title{
Clinical, genetic, and molecular characterization of hyperphosphatasia with mental retardation: a case report and literature review
}

\author{
Layal Abi Farraj, Wassim Daoud Khatoun ${ }^{1}$, Naji Abou Chebel², Victor Wakim¹, Katia Dawalii and \\ Michella Ghassibe-Sabbagh ${ }^{1 *}$ iD
}

\begin{abstract}
Background: Hyperphosphatasia with mental retardation syndrome (HPMRS) is a recessive disorder characterized by high blood levels of alkaline phosphatase together with typical dysmorphic signs such as cleft palate, intellectual disability, cardiac abnormalities, and developmental delay. Genes involved in the glycosylphosphatidylinositol pathway and known to be mutated in HPMRS have never been characterized in the Lebanese population.

Case presentation: Herein, we describe a pair of monozygotic twins presenting with severe intellectual disability, distinct facial dysmorphism, developmental delay, and increased alkaline phosphatase level. Two individuals underwent whole exome sequencing followed by Sanger sequencing to confirm the co-segregation of the mutation in the consanguineous family. A biallelic loss of function mutation in PGAP3 was detected. Both patients were homozygous for the c.203delC (p.C68LfsX88) mutation and the parents were carriers confirming the founder effect of the mutation. High ALP serum levels confirmed the molecular diagnosis.

Conclusion: Our findings have illustrated the genomic profile of PGAP3-related HPMRS which is essential for targeted molecular and genetic testing. Moreover, we found previously unreported clinical findings such as hypodontia and skin hyperpigmentation. These features, together with the novel mutation expand the phenotypic and genotypic spectrum of this rare recessive disorder.
\end{abstract}

Keywords: Cleft palate, PGAP3, Lebanese population, Syndromic, HPMRS

\section{Background}

Orofacial clefts (OFCs) are the most common congenital craniofacial birth defects [10]. Their prevalence varies widely between populations with an average of $1 / 700$ live births $[10,19]$. OFCs arise as a result of genetic and environmental factors interfering abnormally with a set of coordinated events leading to the development of the craniofacial processes between the 4th and 8th gestational weeks [6]. According to genetic and epidemiological risk factors, OFCs have been divided into cleft lip with or without the palate $(\mathrm{CL} / \mathrm{P})$ and cleft palate only

\footnotetext{
* Correspondence: michella.sabbagh@lau.edu.lb

${ }^{1}$ Department of Natural Sciences, School of Arts and Sciences, Lebanese

American University, Beirut, Lebanon

Full list of author information is available at the end of the article
}

(CPO) [9]. Clefts are known for their complex etiology and lifelong morbidity [9]. OFCs are mainly nonsyndromic ( $70 \%$ of $\mathrm{CL} / \mathrm{P}$ and $50 \%$ of $\mathrm{CPO}$ ) [9]. A large number of the genetic factors involved in the remaining $30 \%$ syndromic clefts has been identified through the advancement of the genetic screening techniques $[3,8,23]$. Hyperphosphatasia with mental retardation syndrome (HPMRS) (OMIM \# 239300) was first described in 1970, and was later designated as Mabry Syndrome [4, 17]. Typical facial dysmorphic signs such as cleft palate, intellectual disability, cardiac abnormalities, and brachytelephalangy are frequently described in affected individuals $[1,11,15]$. Elevated serum levels of alkaline phosphatase (ALP) have been recorded in several patients $[1,8]$.

(C) The Author(s). 2019 Open Access This article is distributed under the terms of the Creative Commons Attribution 4.0 International License (http://creativecommons.org/licenses/by/4.0/), which permits unrestricted use, distribution, and 
Characterizing mutations underlying diseases with Mendelian patterns of inheritance by using exome sequencing is a powerful approach to explain the etiology of the disease. As part of a study of the genetic basis of different syndromic cleft phenotypes in the Lebanese population, we report the results of exome sequencing of four individuals from the same family. Our data shows novel variants in the previously established causative gene, PGAP3 (OMIM \#611801): a new phenotypic relationship with HPMRS.

\section{Case presentation}

\section{Data collection}

Since establishing the craniomaxillofacial research project at the Lebanese American University, we have examined over 100 patients with orofacial dysmorphic features, including a pair of twins suffering from a severe developmental syndrome. Participants provided written informed consent prior to filling out the questionnaire or giving blood samples. Parents of the minor participants provided a written declaration of consent as they were legally authorized representatives. Research and data collection were carried out in compliance with the Helsinki Declaration, with the approval of the LAU Institutional Review Board and the Committee on Human Subjects in Research (CHSR). De-identified data about the neurological, muscular, epithelial, and connective tissues related development was collected and all medical problems were obtained from medical charts.

ALP levels were measured in all patients. Information including brain imaging, EEG, skin biopsies, echocardiograms, and thyroid profile were also performed. Parents were examined and genomic DNA was extracted from peripheral blood of the patients and their two parents using the standard phenol-chloroform extraction procedure.

\section{Genetic analysis}

Whole exome enrichment was performed on genomic DNA using the SureSelect Human All Exon V6 (Agilent). Whole exome sequencing was performed on an Illumina Hi-Seq 3000 platform using a $150 \mathrm{bp}$ paired-end protocol with an average depth of 60x. Reads were aligned against the human assembly GRCh37 using Burrows-Wheeler Aligner (BWA). Data analysis was performed using SAMtools [16], Picard [5], the Genome Analysis Toolkit (GATK) [18] and Variant Effect Predictor (VEP). Polymorphisms were removed by reference to their population frequencies by searching for such mutations in genetic disease databases including OMIM (http://omim.org), HGMD (http:// www.hgmd.cf.ac.uk/ac/index.php), ClinVar (https:www.ncbi .nlm.nih.gov/clinvar), the database of the 1000 Genomes Project (http://www.1000genomes.org/), ESP6500 (http:// evs.gs.washington.edu/EVS/), and ExAC (http://exac.broad institute.org). The effects of mutations on protein function were predicted with the aid of PolyPhen 2 (http://genetics. bwh.harvard.edu/pph2/), SIFT (http://sift.jcvi.org), and CADD (https://cadd.gs.washington.edu/). The prioritization of genes was conducted using Pubmatrix (https://pubma trix.irp.nia.nih.gov/) followed by further assessment in relation to clinical characteristics.

\section{Clinical characteristics}

There was a first-degree consanguinity between the parents of the twin pair (Fig. 1). No family history of HPMRS was recorded and both parents reported to be young and healthy at the time of conception. The twins were nineyear-old at the time of data collection. They presented hypotonia with a lack of voluntary coordination of muscle movements prohibiting them from walking, absence of speech, and abnormalities in the eye movement with down-slanting eyelids. They both suffered from growth retardation and epilepsy. No signs of autism or hyperactivity were recorded. Head magnetic resonance imaging showed evidence of $2 \times 2 \mathrm{~cm}$ area of bleed in the left temporal lobe of both affected individuals with the presence of an

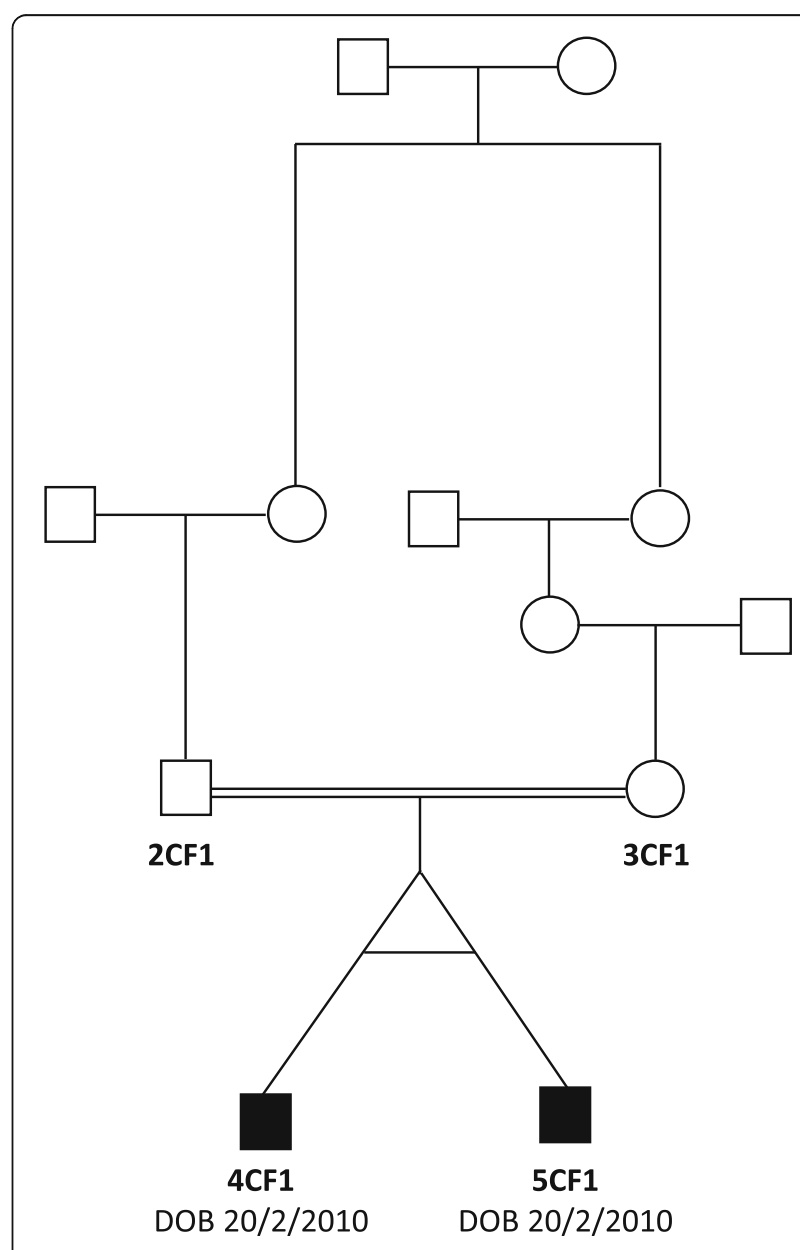

Fig. 1 Pedigree of family. Filled symbols, affected; $\square=0$, consanguinity; $\AA$, monozygotic twins 
abnormal vein. No arterial abnormality was seen. The pattern of gyration and myelination was normal as well as the ventricles. Skin biopsy following the description of skin hyperpigmentation confirmed the diagnosis of incontinentia pigmenti, although the WES data did not show any mutation in IKBKG, previously reported to be linked to the condition [25]. The twins had typical facial features with a wide nasal bridge, tent-shaped lips, and a cleft of the anterior and posterior palate together with typical recurrent ear infections. Gastroscopy in one of the twins, 4CF1, showed the presence of marked esophagitis, large hiatal hernia, petechial gastritis, and minimal nodular duodenitis. In the other twin, 5CF1, cardiac ultrasonography showed a congenital ventricular septal defect with the presence of a heart murmur. He also presented a clinodactyly of the fifth digit (Fig. 1 and Table 1).

\section{Mutational analysis}

The analysis of the whole exome data showed that the patient 5CF1 carried a homozygous frameshift mutation in PGAP3, exon 2, c.203delC (p.C68LfsX88) (Fig. 1). The father (2CF1) was heterozygous for the same mutation. Sanger sequencing was used to confirm the mutation in the two above-studied individuals, and to look for its presence in the mother (3CF1) as well as the other twin (4CF1). Results showed that both parents were carriers for the mutation, and both children were homozygous for the mutation. The presence of an $A>G$ substitution in intron 2, 110 base pairs $3^{\prime}$ of the c.203delC change (genomic position $39,685,888 \mathrm{bp}$ ), excluded the hemizygous mutation hypothesis. Although the cytosine on the position 203 has previously been shown to be substituted, the current deletion has not been reported previously in any population. No other relevant nucleotide changes were found. In order to confirm the presence of the HPMRS syndrome, a dosage of the level of ALP was done. The results showed that the ALP level was of $390 \mathrm{U} / \mathrm{L}$ and $327 \mathrm{U} / \mathrm{L}$ for 4CF1 and 5CF1 respectively.

\section{Structural analysis}

The mutation resulted in a stop codon replacing a cysteine residue at the 68th amino acid position. Thus, the corresponding protein has only 67 amino acids, compared to 320 amino acids in the wild type protein. Phyre2 suggested the 320 amino-acid normal protein to present seven transmembrane alpha helices spanning amino acids 99 to 296 [13]. However, since the mutated protein has only the first 67 amino acids, no transmembrane helices in this protein were suggested by Phyre2 [13]. This indicates that the protein is no longer capable of getting integrated in the membrane of Golgi apparatus, as the literature states [22]. The templates to which both the normal and the mutated proteins were aligned in the Phyre2 software further support this hypothesis (Fig. 2).

\section{Discussion and conclusions}

In the 2001 version of the London Dysmorphology Database, 487 monogenic syndromes have been reported to be associated with ORFs [7]. As part of the effort of studying the genetic basis of Lebanese families presenting syndromic clefts, we were able to identify a novel PGAP3, "post-GPI attachment top 3", mutation in one consanguineous family. The latter mutation suggested

Table 1 Summary of the Clinical data comparing previously reported cases of PAPG3 with our two current cases

\begin{tabular}{lll}
\hline Clinical Data & Current case report & Previous Cases Reported (26) [1, 20, 24] \\
\hline Consanguinity & $2 / 2$ & $16 / 26$ \\
Cleft Palate & $2 / 2$ & $17 / 26$ \\
Postnatal Microcephaly & $2 / 2$ & $8 / 26$ \\
Short Stature & $2 / 2$ & $2 / 26$ \\
Seizures & $2 / 2$ & $17 / 26$ \\
Coarse Facies & $2 / 2$ & $26 / 26$ \\
Developmental Delay & $2 / 2$ & $26 / 26$ \\
Speech Delay & $2 / 2$ & $26 / 26$ \\
High ALP level (relative to age) & $2 / 2$ & $26 / 26$ \\
Hyperactive \& Autistic Behavior & $2 / 2$ & $14 / 26$ \\
Congenital Heart Defects & $1 / 2$ & $2 / 26$ \\
Clinodactyly & $2 / 2$ & $0 / 26$ \\
Small teeth & $0 / 2$ & $1 / 26$ \\
Hypodontia & $2 / 2$ & $0 / 26$ \\
Hyperpigmentation(Incontinentia Pigmenti) & $2 / 2$ & $0 / 26$ \\
\hline
\end{tabular}

In bold font are the new clinical features that were not previously reported in any case of the mutation 


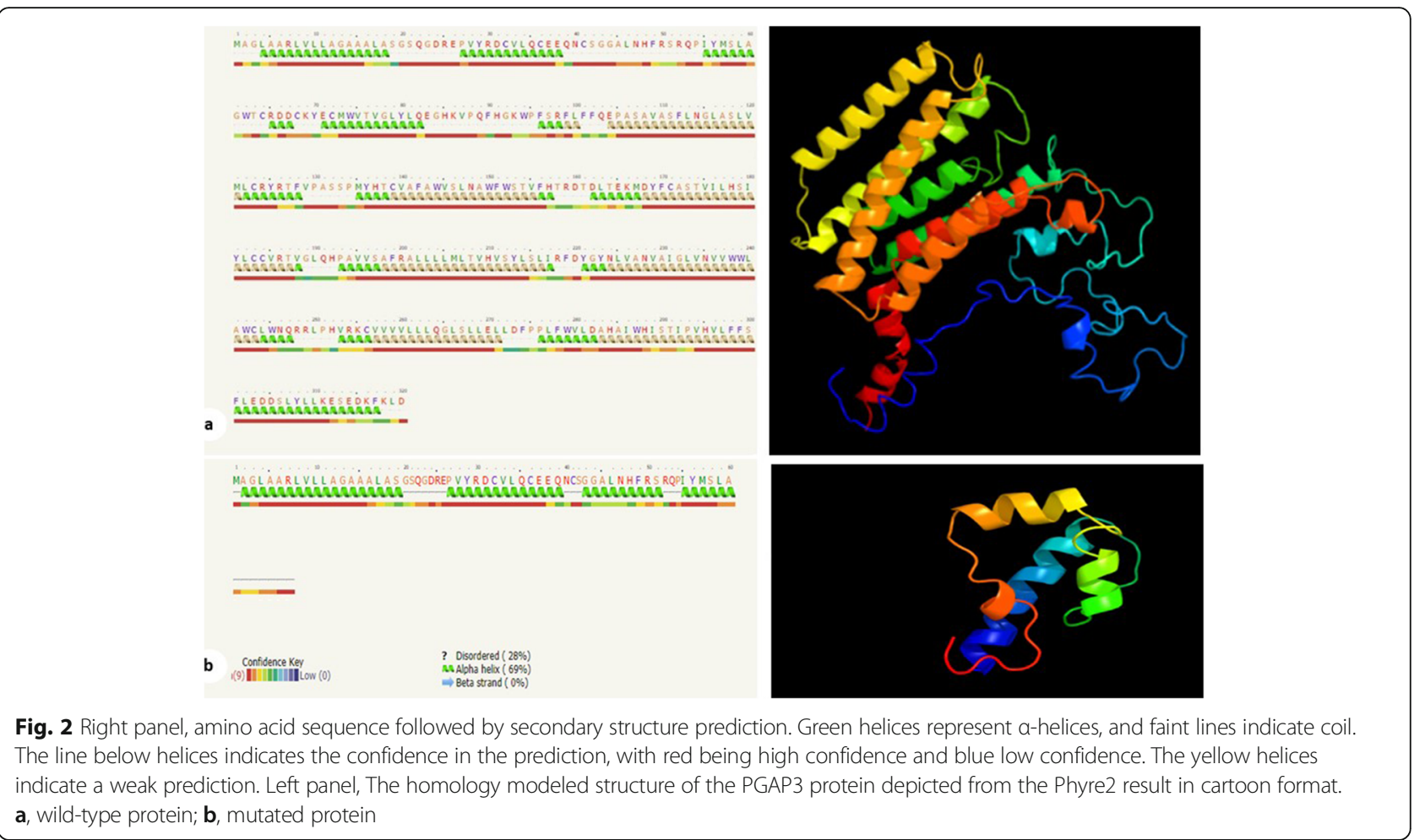

that the family presented a hyperphosphatasia with mental retardation syndrome, falling under the monogenic syndrome categorization. In order to confirm that the dysmorphic features encountered in the family are part of the HPMRS syndrome, a dosage of the level of ALP was done. The elevated levels of ALP in the blood confirmed the molecular diagnosis in the family. Interestingly, the level of ALP is 1.03 and 1.24 higher than the normal ALP range for boys between 7 and 9 years (86-315 U/L).

PGAP3 encodes a glycosylphosphatidylinositol (GPI)specific phospholipase that removes unsaturated fatty acids from GPI anchored protein in the Golgi apparatus where it primarily localizes and performs the first step of fatty acid remodeling [22]. The GPI-anchored proteins (GPI-APs) and lipid rafts proper association is mainly due to the remodeling of the constituent fatty acids on GPI. The symptoms caused by PGAP3 mutations could be caused by the inability of the GPI-APs in cells to localize on lipid rafts because of their non-remodeled lipid structure, despite the presence of normal levels of PGAP3 in the cells [14].

To date, only 16 pathogenic PGAP3 mutations have been identified in patients with HPMRS $[1,2,12,14,20$, $21,24,26]$. Ten out of the 16 mutations are in exon 3 and exon 7 which are considered hotspots for mutations. The majority of these mutations are missense mutations; however, frameshift, splice site, and 3'UTR mutations were reported. The mutation reported in this case report raises the number of mutations to 17 mutations. It is a homozygous frameshift mutation located in exon 2, c.203delC (p.C68LfsX88), affecting monozygotic twins resulting from a consanguineous marriage.

The monozygotic twins have features concordant with an HPMRS phenotype although they showed the most severe spectrum of the phenotype. In addition to signs already associated with HPMRS, they also showed clinodactyly, hypodontia, as well as hyperpigmentation, a phenotype that has never been reported in HPMRS caused by a PGAP3 mutation (Table 1).

In summary, we reported a novel homozygous mutation in PGAP3 in a consanguineous Lebanese cleft syndromic family associated with hyperphosphatasia with mental retardation syndrome. Novel features not reported as part of this syndrome before were present. This case report shows that ORFs associated with global developmental delay and facial dysmorphism with elevated alkaline phosphatase are important clues for HPMRS. In addition, it shows that whole exome sequencing helps increase the diagnostic yield for heterogeneous conditions such as syndromic ORFs encountered more commonly in consanguineous families. Molecular diagnosis might enable a proper recognition of the associated syndromes and anomalies with ORFs by finding the causative agents, which is essential for informative genetic counselling, prenatal diagnosis, targeted treatment and prevention in case it is possible. 


\section{Abbreviations}

ALP: Alkaline phosphatase; CHSR: Committee on Human Subjects in Research: CPO: Cleft palate only; GPI: Glycosylphosphatidylinositol; GPI-Aps: GPI-anchored proteins; HPMRS: Hyperphosphatasia with mental retardation syndrome; OFCs: Orofacial clefts; WES: Whole Exome Sequencing

\section{Acknowledgements}

The authors are grateful to the patients for their participation in the study.

\section{Authors' contributions}

LAF did the whole exome sequencing and analysis. WDK did the protein remodeling analysis. NAC contributed to the diagnoses and data collection. WW assisted in patient selection and table preparation. KD did the Sanger sequencing. MGS guided the whole study, drafted, and revised the manuscript. All authors read and approved the final manuscript.

\section{Funding}

This study was partially funded by the Lebanese American University, School Research and Development Council (SRDC), Research Grant: "Genetic Determinants of Craniofacial Anomalies".

\section{Availability of data and materials}

The datasets generated and/or analyzed in this case report are available from the corresponding author upon reasonable request.

\section{Ethics approval and consent to participate}

The study was approved by the LAU Institutional Review Board and the Committee on Human Subjects in Research (CHSR) (approval no. LAU.SAS.MS1.23). Informed consent was obtained from participants. All experiments were carried out in accordance with relevant guidelines and regulations.

\section{Consent for publication}

Not applicable

\section{Competing interests}

The authors declare that they have no competing interests.

\section{Author details}

'Department of Natural Sciences, School of Arts and Sciences, Lebanese American University, Beirut, Lebanon. ${ }^{2}$ School of Medicine, Lebanese American University, Beirut, Lebanon.

Received: 25 August 2019 Accepted: 10 October 2019

Published online: 04 November 2019

\section{References}

1. Abdel-Hamid MS, Issa MY, Otaify GA, et al. PGAP3-related hyperphosphatasia with mental retardation syndrome: report of 10 new patients and a homozygous founder mutation. Clin Genet. 2018;93(1):84-91.

2. Abouelhoda M, Sobahy T, El-Kalioby M, et al. Clinical genomics can facilitate countrywide estimation of autosomal recessive disease burden. Genet Med. 2016;18(12):1244-9.

3. Basha M, Demeer B, Revencu N, et al. Whole exome sequencing identifies mutations in $10 \%$ of patients with familial non-syndromic cleft lip and/or palate in genes mutated in well-known syndromes. J Med Genet. 2018; 55(7):449-58.

4. Cole DE, Thompson MD. Neurogenetic Aspects of Hyperphosphatasia in Mabry Syndrome. Subcell Biochem. 2015;76:343-61.

5. DePristo MA, Banks E, Poplin $\mathrm{R}$, et al. A framework for variation discovery and genotyping using next-generation DNA sequencing data. Nat Genet. 2011:43(5):491-8

6. Ferguson MW. Palate development. Development. 1988;103:41-60.

7. Fryns JP, de Ravel TJ. London Dysmorphology Database, London Neurogenetics Database and Dysmorphology Photo Library on CD-ROM [Version 3] 2001R. M. Winter, M. Baraitser, Oxford University Press, ISBN 019851-780, pound sterling 1595. Hum Genet. 2002:111(1):113.

8. Fu L, Liu Y, Chen Y, Yuan Y, Wei W. Mutations in the PIGW gene associated with hyperphosphatasia and mental retardation syndrome: a case report. BMC Pediatr. 2019;19(1):68
9. Ghassibe-Sabbagh M, Desmyter L, Langenberg T, et al. FAF1, a gene that is disrupted in cleft palate and has conserved function in zebrafish. Am J Hum Genet. 2011;88(2):150-61.

10. Ghassibe M, Bayet B, Revencu N, et al. Orofacial clefting: update on the role of genetics. B-ENT. 2006;2(Suppl 4):20-4

11. Horn D, Schottmann G, Meinecke P. Hyperphosphatasia with mental retardation, brachytelephalangy, and a distinct facial gestalt: delineation of a recognizable syndrome. Eur J Med Genet. 2010;53(2):85-8.

12. Howard MF, Murakami Y, Pagnamenta AT, et al. Mutations in PGAP3 impair GPI-anchor maturation, causing a subtype of hyperphosphatasia with mental retardation. Am J Hum Genet. 2014:94(2):278-87.

13. Kelley LA, Mezulis S, Yates CM, Wass MN, Sternberg MJ. The Phyre2 web portal for protein modeling, prediction and analysis. Nat Protoc. 2015;10(6):845-58

14. Knaus A, Awaya T, Helbig I, et al. Rare noncoding mutations extend the mutational Spectrum in the PGAP3 subtype of Hyperphosphatasia with mental retardation syndrome. Hum Mutat. 2016;37(8):737-44.

15. Krawitz PM, Schweiger MR, Rodelsperger C, et al. Identity-by-descent filtering of exome sequence data identifies PIGV mutations in hyperphosphatasia mental retardation syndrome. Nat Genet. 2010; 42(10):827-9.

16. Li H, Handsaker B, Wysoker A, et al. The sequence alignment/map format and SAMtools. Bioinformatics. 2009;25(16):2078-9.

17. Mabry CC, Bautista A, Kirk RF, et al. Familial hyperphosphatase with mental retardation, seizures, and neurologic deficits. J Pediatr. 1970;77(1):74-85.

18. McKenna A, Hanna M, Banks E, et al. The genome analysis toolkit: a MapReduce framework for analyzing next-generation DNA sequencing data. Genome Res. 2010;20(9):1297-303.

19. Mossey PA, Little J, Munger RG, Dixon MJ, Shaw WC. Cleft lip and palate. Lancet. 2009;374(9703):1773-85.

20. Nampoothiri S, Hebbar M, Roy AG, et al. Hyperphosphatasia with mental retardation syndrome due to a novel mutation in PGAP3. J Pediatr Genet. 2017:6(3):191-3.

21. Pagnamenta AT, Murakami Y, Taylor JM, et al. Analysis of exome data for 4293 trios suggests GPI-anchor biogenesis defects are a rare cause of developmental disorders. Eur J Hum Genet. 2017;25(6):669-79.

22. Pei J, Millay DP, Olson EN, Grishin NV. CREST--a large and diverse superfamily of putative transmembrane hydrolases. Biol Direct. 2011;6:37.

23. Pengelly RJ, Upstill-Goddard R, Arias $L$, et al. Resolving clinical diagnoses for syndromic cleft lip and/or palate phenotypes using whole-exome sequencing. Clin Genet. 2015;88(5):441-9.

24. Sakaguchi T, Zigman T, Petkovic Ramadza D, et al. A novel PGAP3 mutation in a Croatian boy with brachytelephalangy and a thin corpus callosum. Hum Genome Var. 2018:5:18005.

25. Swinney CC, Han DP, Karth PA. Incontinentia Pigmenti: a comprehensive review and update. Ophthalmic Surg Lasers Imaging Retina. 2015:46(6):650-7.

26. Yavarna T, Al-Dewik N, Al-Mureikhi M, et al. High diagnostic yield of clinical exome sequencing in middle eastern patients with Mendelian disorders. Hum Genet. 2015;134(9):967-80.

\section{Publisher's Note}

Springer Nature remains neutral with regard to jurisdictional claims in published maps and institutional affiliations.

Ready to submit your research? Choose BMC and benefit from:

- fast, convenient online submission

- thorough peer review by experienced researchers in your field

- rapid publication on acceptance

- support for research data, including large and complex data types

- gold Open Access which fosters wider collaboration and increased citations

- maximum visibility for your research: over $100 \mathrm{M}$ website views per year

At BMC, research is always in progress.

Learn more biomedcentral.com/submission 